Article

\title{
Changes in Beneficial $C$-glycosylflavones and Policosanol Content in Wheat and Barley Sprouts Subjected to Differential LED Light Conditions
}

\author{
Muthusamy Muthusamy ${ }^{1,+}{ }^{\circledR}$, Jong Hee Kim ${ }^{1,2,+}$, Suk Hee Kim ${ }^{1}$, Joo Yeol Kim ${ }^{1}$, \\ Jeong Wook Heo ${ }^{3}$, HanGyeol Lee ${ }^{4}$, Kwang-Sik Lee ${ }^{4}$, Woo Duck Seo ${ }^{4}$ (D), Soyoung Park ${ }^{1}$, \\ Jin A Kim ${ }^{1}$ and Soo In Lee ${ }^{1, *(D)}$ \\ 1 Department of Agricultural Biotechnology, National Institute of Agricultural Sciences (NAS), RDA, \\ Jeonju 54874, Korea; biotech.muthu@gmail.com (M.M.); gllmon@naver.com (J.H.K.); \\ hbhb0@naver.com (S.H.K.); rlawnduf@korea.kr (J.Y.K.); psy0203@korea.kr (S.P.); jakim72@korea.kr (J.A.K.) \\ Division of Horticultural Biotechnology, Hankyung National University, Anseong 17579, Korea \\ 3 Department of Agricultural Engineering, National Institute of Agricultural Sciences (NAS), RDA, \\ Jeonju 54874, Korea; wooncho@korea.kr \\ 4 Division of Crop Foundation, National Institute of Crop Science (NICS), RDA, Wanju 55365, Korea; \\ gajae93@gmail.com (H.L.); kslee840118@gmail.com (K.-S.L.); swd2002@korea.kr (W.D.S.) \\ * Correspondence: silee@korea.kr; Tel.: +82-63-238-4618; Fax: +82-63-238-4604 \\ + These authors contributed equally to this work.
}

Received: 24 September 2020; Accepted: 4 November 2020; Published: 6 November 2020

\begin{abstract}
The spectral quality and intensity of light, photoperiodism, and other environmental factors have profound impacts on the metabolic composition of light-dependent higher plants. Hence, we investigate the effects of fluorescent light $\left(96 \mu \mathrm{mol} \mathrm{m}^{-2} \mathrm{~s}^{-1}\right)$ and white $\left(100 \mu \mathrm{mol} \mathrm{m}^{-2} \mathrm{~s}^{-1}\right)$, blue $\left(100 \mu \mathrm{mol} \mathrm{m}{ }^{-2} \mathrm{~s}^{-1}\right)$, and red $\left(93 \mu \mathrm{mol} \mathrm{m}^{-2} \mathrm{~s}^{-1}\right)$ light-emitting diode (LED) light irradiation on the C-glycosylflavone and policosanol contents in young seedlings of wheat and barley. Ultra-high-performance liquid chromatography (UHPLC) analyses of $C$-glycosylflavone contents in barley reveal that the saponarin content is significantly enhanced under blue LED light irradiation. Under similar conditions, isoorientin and isoschaftoside contents are improved in wheat seedlings. The contents of these $C$-glycosylflavones differed along with the light quality and growth period. The highest accumulation was observed in sprouts after three days under blue LED light irradiation. GC/MS analyses of policosanol contents showed that 1-hexacosanol $(\mathrm{C} 26: \mathrm{o}-\mathrm{OH})$ in barley and 1-octacosanol $(\mathrm{C} 28: \mathrm{o}-\mathrm{OH})$ in wheat seedlings were reduced under LED light irradiation, compared to seedlings under fluorescent light conditions. Nonetheless, the policosanol contents gradually improved with the extension of growth times and treatments, irrespective of the light quality. Additionally, a positive correlation was observed between the expression pattern of biosynthesis-related genes and the respective metabolite content in barley. This study demonstrates that blue LED light irradiation is useful in maximizing the $C$-glycosylflavone content in barley and wheat sprouts.
\end{abstract}

Keywords: saponarin; isoorientin; hexacosanol; octacosanol; fatty acyl-coenzyme A reductase (FAR)

\section{Introduction}

Wheat (Triticum aestivum L.) is one of the staple food grains for approximately $40 \%$ of the global population [1]. It ranks third in terms of global production and its nutritional importance in the human diet has long been investigated [2]. Similarly, barley (Hordeum vulgare L.) is the fourth most important cereal crop for both humans and animals worldwide, having the highest 
dietary fiber content $[3,4]$. Barley is rich in biologically active molecules/metabolites, which are essential for plants. These metabolites have the potential to exhibit health benefits in the human diet. Barely or its extracts have shown powerful antioxidant effects as dietary supplements for humans. These antioxidant effects are mainly attributable to the presence of saponarin, lutonarin, and hexacosanol molecules [3]. Barley grass also possesses numerous other phytonutrients, including gamma-aminobutyric acid (GABA), flavonoids, proteins, minerals, pigments, vitamins (A, B1, $\mathrm{C}$, and E), dietary fiber, polysaccharides, alkaloids, and polyphenols [4]. Recent reports discussing the broad therapeutic roles of functional ingredients or derived components of barley suggest that it may be the best fit in the modern human diet as a functional food [5-7]. Barley saponarin has several health benefits, including anti-inflammatory response $[4,8]$, prevention of bacterial infections [9], regulation of glucose homeostasis, insulin sensitivity [10], reducing low-density lipoprotein (LDL) cholesterol [4], and anti-carcinogenic responses [11]. Similarly, isoorientin from wheat acts as an anti-cancer compound [12] and also possesses anti-inflammatory, antibacterial, antiviral, antiplatelet [13], and antioxidant activities [14]. Wheat (or its derived products) also possesses several beneficial bioactive molecules, including pelargonidin and cyanidin derivatives [15], essential amino acids, fatty acids, flavonoids (e.g., rutin, quercetin, and catechin), vitamin C [16], and policosanols [17]. The $C$-glycosylflavone and policosanol content in barley vary with growth duration [18]. Reports have claimed that sprouts produce higher concentrations of health-promoting molecules than grains [19].

Sprouting implies a series of active biochemical, metabolic, and physiological processes, resulting in the release of active nutrients (e.g., free amino acids and lipid catabolism) for growing plant tissues $[18,19]$. These metabolites often possess potential health benefits for humans $[18,20]$. Thus, sprouting is considered one of the easiest natural strategies to enhance nutritional profiles with healthy attributes [20]. Owing to their nutritive values, sprouting seeds has recently received growing interest. Meanwhile, researchers have attempted to identify the presence of novel functional ingredients of sprouts under varying growth and environmental conditions [18,19]. Plants increase the production of a variety of metabolites, in order to mitigate the effects of adverse environmental factors, such as drought [21], salinity [22], high-intensity light or artificial lighting [23], temperature [24], and elevated $\mathrm{CO}_{2}$ levels [25]. Therefore, effective management and/or the controlled application of physical energy forms (e.g., light, temperature, and water) may serve as a viable option to enhance the accumulation of health-promoting compounds in sprouts, which has been shown to be successful in previous attempts [25-27]. In terms of physical energy forms, light irradiation has been employed in different sprouting seeds, in order to increase metabolites with health-promoting benefits $[23,28]$. The availability of artificial lighting resources (e.g., light-emitting diodes (LEDs)) renders the possibility of studying the effects of specific light on the concentrations of biologically important metabolites in plants. Herein, the potential effects of a fluorescent lamp (FL) and different spectra of LED light irradiations (white, blue, and red) on beneficial metabolite content were investigated in barley and wheat sprouts. Additionally, we attempted to identify and profile the expression patterns of metabolite biosynthesis-related genes of barley sprouts. This study facilitates understanding of the differential responses relating to $C$-glycosylflavones and policosanols in wheat and barley sprouts.

\section{Results}

\subsection{Changes of C-glycosylflavone Content in Barley and Wheat Seedlings Exposed to Differential LED Light Irradiation}

The changes in saponarin (barley), isoorientin, and isoschaftoside (wheat) content in sprouts treated with different light qualities (FL and white, blue, or red LED irradiation) were measured using ultra-high-performance liquid chromatography (UHPLC) in plant materials harvested after 3-9 days of treatment. The UHPLC results of barley and wheat sprouts revealed that blue LED light irradiation increased the $C$-glycosylflavone content more than other light conditions. The LED irradiation differentially influenced the saponarin content in the barley sprouts. In comparison with FL, LED light irradiation significantly altered the content: Blue light irradiation prominently improved 
the saponarin content (51.7\%-57.7\%) across all growth stages and irradiation periods (Figure 1A). The highest concentration of saponarin was observed in sprouts after 3 days of blue LED light irradiation. Interestingly, sprouts treated with red LED light irradiation demonstrated a significant reduction in saponarin content, compared to their respective controls, after 3, 5, 7, and 9 days of irradiation. Conversely, white LED irradiation for three consecutive days did not alter the content; however, on day 5 , it statistically significantly increased the contents. The extension of white LED treatment for 7 or 9 days resulted in a reduction of saponarin content. Moreover, regardless of the lighting resource or quality, a consistent reduction in saponarin content was observed in the growing sprouts. Among the growth times and light qualities tested in this study, the highest content of saponarin was observed in blue LED irradiated sprouts on the 3rd day, while red LED radiation remarkably reduced the content in all treatments and sprout growth periods.
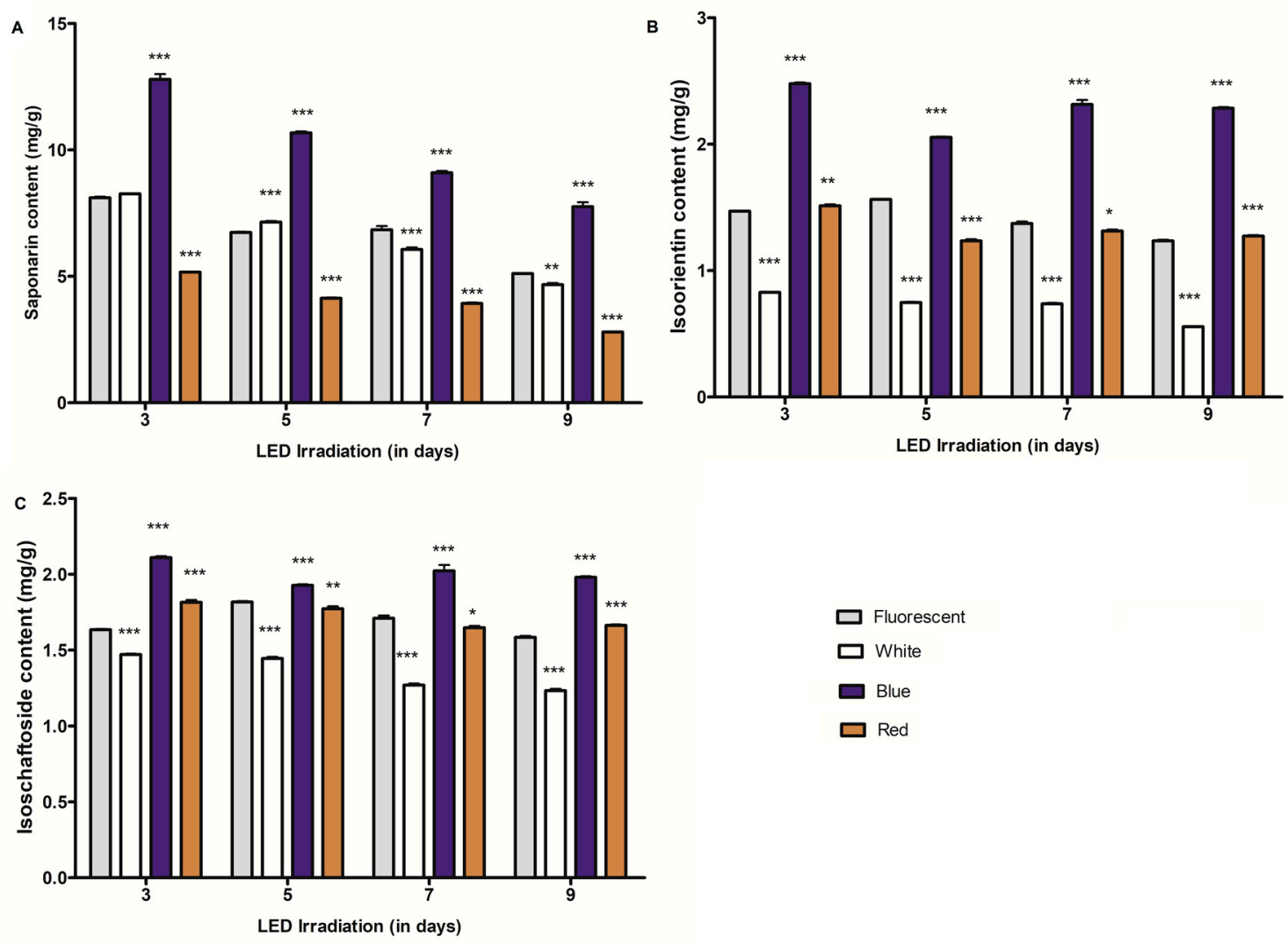

Figure 1. C-glycosylflavone content in young barley and wheat seedlings subjected to differential light qualities. (A) represents the saponarin content of barley sprouts (mg/g dry weight (DW)) under different light and growth periods, while $(\mathbf{B}, \mathbf{C})$ represent the isoorientin and isoschaftoside contents (mg/g DW), respectively, of wheat sprouts. ${ }^{*}(p<0.05),{ }^{* *}(p<0.001)$, and ${ }^{* * *}(p<0.0001)$ indicate the statistical significance.

Isoschaftoside and isoorientin are the major flavone-C-glycosides ( $C$-glycosylflavones) frequently reported in wheat and its derived products [29]. In this study, we found that these metabolites were significantly altered in wheat sprouts exposed to LED light irradiation over 3-9 days (Figure 1B,C). LED light irradiation significantly altered the metabolite concentration in barley and wheat sprouts, compared to their content in sprouts treated with traditional fluorescent lamp light conditions. Blue LED light irradiation markedly improved the concentration of isoschaftoside as well as isoorientin, compared to control (FL) or other (white and red) LED treatments. The highest mean concentrations of 2.1 and $2.47 \mathrm{mg}$ (per g dry weight (DW)) of isoschaftoside and isoorientin, respectively, were observed in seedlings subjected to 3 days of blue LED light irradiation. Under similar conditions, the seedlings treated with FL accumulated $1.63 \mathrm{mg}$ and $1.46 \mathrm{mg}$ (per g DW) of isoschaftoside and isoorientin, 
respectively (Figure 1B,C). On day 3, a slight improvement in isoschaftoside (11.04\%) and isoorientin $(2.78 \%)$ content was also observed in red LED light irradiated sprouts. However, 5 and 7 days of red LED light irradiation significantly reduced the isoorientin and isoschaftoside contents in sprouts. Compared to FL, a maximum of $21.15 \%$ reduction in isoorientin content was observed after five days of red LED light irradiation. Under similar conditions, a 2.25\% reduction was noted for isoschaftoside content (Figure 1C). Conversely, 9 days of red light irradiation increased the isoorientin $(3.15 \%)$ and isoschaftoside $(5.06 \%)$ contents in sprouts. Compared to FL, white LED light irradiation led to reductions in the isoschaftoside (9.81-22.1\%) and isoorientin (43.84-55.3\%) levels across all growth times. In terms of sprout growth periods, the highest accumulation of metabolites was observed after 3 days of light treatment (Figure 1 and Figure S1).

\subsection{Effect of LED Light Irradiation on Major Long-Chain Fatty-Alcohol (Policosanol) Biosynthesis in Barley and Wheat Seedlings}

Crude extracts comprised of policosanols from barley and wheat sprouts grown under FL, white, blue, and red LED light irradiations for 3-9 days were profiled and quantified by gas chromatography coupled with mass spectroscopy (GC/MS). GC/MS results of policosanol content in barley and wheat sprouts indicated that growth time was an influential factor in determining the hexacosanol and octacosanol content in these sprouts. Hexacosanol, a major constituent of the policosanol profile of barley seedlings, was altered during LED light irradiation in accordance with sprout growth periods. In general, the hexacosanol content ( $\mu \mathrm{g} / \mathrm{g} \mathrm{DW})$ in LED light treated samples was significantly lower than that in seedlings treated for 3 or 9 days with FL (Figure 2A). The comparative analysis of hexacosanol content between FL and LED irradiation at the third day of treatment showed that hexacosanol content was significantly reduced for white and blue LED light irradiations. Under these conditions, a maximum reduction of $43.3 \%$ was noted for blue LED light irradiation, while white light treatment showed a reduction of $36.9 \%$ in hexacosanol content. Conversely, on days 5 and 7 , white and blue LED light irradiation did not alter the hexacosanol concentration significantly, in comparison with that of control sprouts. Altogether, the results indicated that the changes in hexacosanol content were not consistent with light qualities, indicating the light quality is not the only factor influencing changes in hexacosanol biosynthesis. Interestingly, hexacosanol content in sprouts treated with either white or blue LED irradiation for 5, 7, and 9 days was higher than the hexacosanol content in sprouts treated only for three days under similar conditions. Likewise, the octacosanol content $(\mu \mathrm{g} / \mathrm{g} D W)$ in wheat seedlings was analyzed using GC/MS (Figure S2). The results showed that the octacosanol content was altered irregularly by different LED light qualities (Figure 2B). A comparative analysis of FL and LED treatments indicated that blue LED light irradiation for 3 and 9 days enhanced the octacosanol content in wheat sprouts. In comparison with FL, white light irradiation also increased the content in sprouts exclusively on day 3. Conversely, red LED irradiation resulted in octacosanol content reduction (on day 5). Nonetheless, the magnitude of change in content was not consistent for any of the LED treatments.

\subsection{Effect of Growth Periods on C-glycosylflavones and Policosanol Content in Young Barley and Wheat Seedlings}

To understand the effect of sprout growth periods (3-9 days) on the metabolite content (saponarin, isoorientin, isoschaftoside, hexacosanol, and octacosanol) under fluorescent and LED light irradiation, we conducted a further investigation. Of all the growth times, the saponarin content of barley and isoorientin/isoschaftoside contents of wheat seedlings were found to be the highest on the 3rd day of the treatment (Figure S3A-C). The contents of these $C$-glycosylflavones showed a declining trend with an increase in growth times (i.e., after 5, 7, and 9 days) in barley and wheat seedlings (Figure S3A-C). However, the magnitude of reduction in metabolite content varied with different light qualities and metabolites in these sprouts. The saponarin content of barley was reduced by $37.96 \%, 43.58 \%, 39.43 \%$, and $45.93 \%$ for FL, white, blue, and red LED light irradiation, respectively, after the further growth 
period of 9 days. A statistically significant negative correlation ( -0.93 to -0.99$)$ between saponarin content and sprout growth period was observed during FL and LED light (white, blue, and red) irradiation (Table 1). A similar attempt to establish relationships between isoorientin content and growth periods in wheat sprouts under FL and LED treatment showed that isoorientin accumulation was negatively correlated with growth periods under specific light (FL, white, and red) treatments. Furthermore, isoschaftoside accumulation is negatively regulated under white and red LED light treatments across growth periods. Nonetheless, their relationships in blue LED irradiated sprouts were inconclusive.
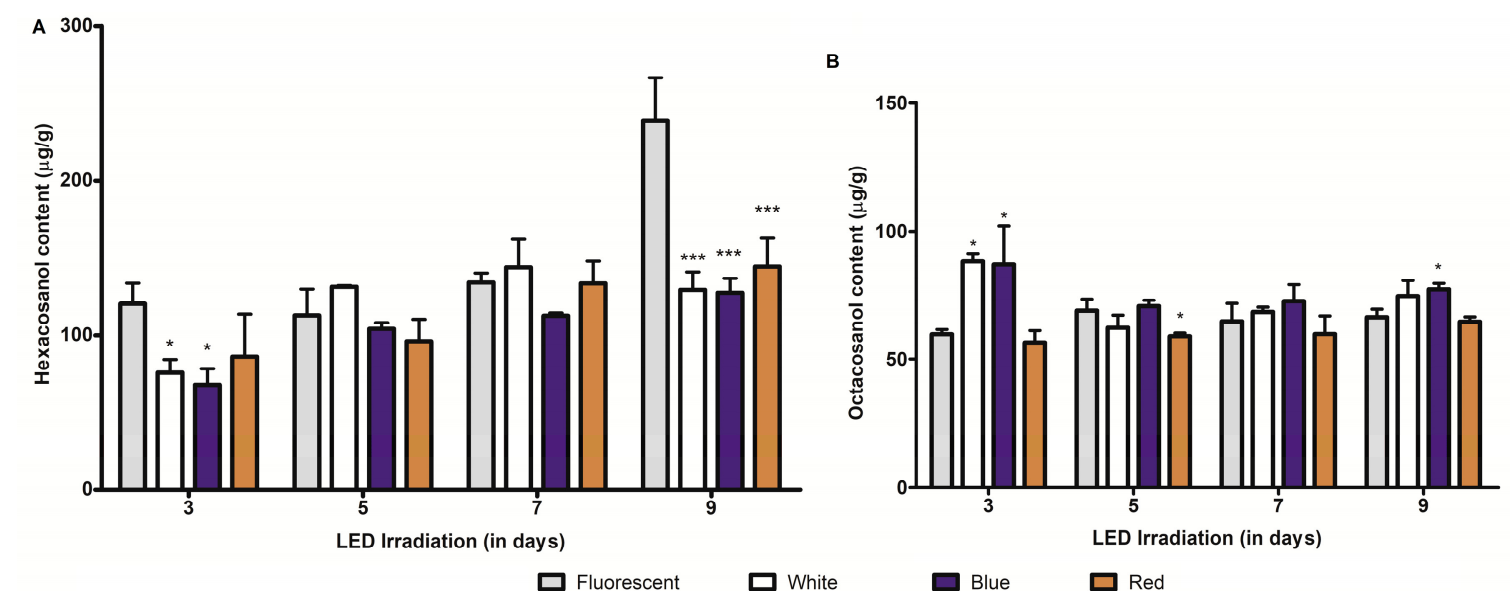

Figure 2. Policosanol content $(\mu \mathrm{g} / \mathrm{g} \mathrm{DW})$ in barley and wheat seedlings during different growth periods and light conditions. (A) denotes hexacosanol (major policosanol) content in barley sprouts, whereas (B) denotes octacosanol content in wheat sprouts. ${ }^{*}(p<0.05)$, and ${ }^{* * *}(p<0.0001)$ indicate the statistical significance.

Table 1. Analysis of correlation statistics (Pearson correlation) among metabolite content, growth periods, and their respective biosynthesis-related gene expression patterns across different light qualities.

\begin{tabular}{|c|c|c|c|c|c|c|c|c|}
\hline & \multicolumn{8}{|c|}{ Metabolite Content vs. Gene Expression } \\
\hline & HvOGT1 & HvFNSII & HvCHS1 & HvFAR2 & HvFAR3 & HvFAR4 & HvFAR5 & HvFAR6 \\
\hline Saponarin & $\begin{array}{c}0.68 \\
(p=0.01)^{* *}\end{array}$ & $\begin{array}{c}-0.53 \\
(p=0.07)\end{array}$ & $\begin{array}{c}0.13 \\
(p=0.68)\end{array}$ & - & - & - & - & - \\
\hline Hexacosanol & - & - & - & $\begin{array}{c}-0.59 \\
(p=0.04) *\end{array}$ & $\begin{array}{c}0.67 \\
(p=0.01)^{* *}\end{array}$ & $\begin{array}{c}-0.62 \\
(p=0.03) \text { * }\end{array}$ & $\begin{array}{c}-0.76 \\
(p=0.004) * *\end{array}$ & $\begin{array}{c}-0.69 \\
(p=0.01)^{* *}\end{array}$ \\
\hline
\end{tabular}

\begin{tabular}{lcccc}
\hline \multicolumn{5}{c}{ Growth periods (-9 days) vs. metabolite content } \\
\hline Fluorescent & White & Blue & Red \\
\hline Saponarin & $-0.93(p=0.00001)^{* * * *}$ & $-0.99(p=0.00001)^{* * * *}$ & $-0.99(p=0.00001)^{* * * *}$ & $-0.97(p=0.00001)^{* * * *}$ \\
\hline Isoorientin & $-0.82(p=0.001)^{* * *}$ & $-0.92(p=0.00002)^{* * * *}$ & $-0.23(p=0.47)$ & $-0.66(p=0.01)^{* *}$ \\
\hline Isoschaftoside & $-0.32(p=0.31)$ & $-0.94(p=0.00001)^{* * * *}$ & $-0.47(p=0.12)$ & $-0.90(p=0.00006)^{* * * *}$ \\
\hline Hexacosanol & $0.79(p=0.001)^{* * *}$ & $0.69(p=0.01)^{* *}$ & $0.91(p=0.00004)^{* * * *}$ & $0.81(p=0.001)^{* * *}$ \\
\hline Octacosanol & $-0.07(p=0.82)$ & $-0.43(p=0.16)$ & $-0.17(p=0.57)$ & $-0.04(p=0.90)$ \\
\hline
\end{tabular}

${ }^{*} p<0.05,{ }^{* *} p<0.001,{ }^{* * *} p<0.0001,{ }^{* * * *} p<0.00001$ indicates statistical significance (replicates were included for measuring Pearson correlation).

In contrast to C-glycosylflavone content, the hexacosanol content in barley sprouts gradually increased with the extension of the growth period. In fact, the highest accumulation of hexacosanol was observed in barley sprouts after 9 days of FL and LED light (white, blue, and red) irradiation treatments (Figure S3D). The correlation coefficient (Pearson) analysis showed that positive correlations (0.69 to 0.91 ) existed between hexacosanol content and growth period, which was statistically significant in both FL and LED irradiated seedlings (Table 1). Unlike hexacosanol, the positive relationship between octacosanol content and wheat growth period was not clear (Figure S3E). As indicated in Figure S3E, 
the growth period did not impose any significant effect on octacosanol content under white, red, and blue LED or FL light irradiation. Nonetheless, a comparison of growth periods under white LED irradiation showed significant differences in octacosanol concentration in wheat sprouts.

2.4. Expression Profiling of Potential Genes Involved in the Biosynthesis of C-glycosylflavones/Flavonoids and Policosanols in Barley Seedlings under Different Light Conditions and Growth Times

The homology-based gene identification approach was used to predict the potential genes associated with the biosynthesis of flavones/flavonoids and long-chain fatty alcohols in the barley genome. The mRNA sequences of potential candidate genes were designated according to their highest sequence similarity to known genes of other crops (Table S1). As shown in Figure S3, sprout growth periods were one of the factors that greatly influenced the saponarin and hexacosanol contents; thus, we selected the growth periods associated with high accumulation of these metabolites for investigation of expression analyses of flavonoid and fatty alcohol biosynthesis-related genes. The expression levels of HvCHS1, HvFNSII, and HvOGT1 for flavonoid biosynthesis and HvFAR2, HvFAR3, HvFAR4, HvFAR5, and HvFAR6 for policosanol biosynthesis were significantly altered under FL, white, blue, and red light irradiation (Figure 3A-H). The expression pattern of $\mathrm{HvOGT1}$ under differential light conditions was positively correlated (0.68) with saponarin content in barley (Table 1). In terms of light responses, the expression of FNSII was significantly upregulated in red LED irradiated seedlings, while its expression was downregulated by white LED light, in comparison with FL or blue LED light. The light-responsive expression of CHS1 was slightly changed under LED irradiation, but the changes were negligible (Figure 3C). In terms of policosanol biosynthesis-related gene expression, the expression pattern of $H v F A R 3$ had a positive correspondence (0.67) with hexacosanol accumulation (Table 1). The HvFAR3 expression was reduced under white and blue LED light irradiation, while it was unaltered in red LED light irradiated seedlings. Interestingly, the expression patterns of HvFAR2, HvFAR4, HvFAR5, and HvFAR6 were negatively correlated (-0.59 to -0.76) with hexacosanol content in barley sprouts. Their expressions were upregulated by one or more LED light irradiations. HvFAR2 and HvFAR5 showed their highest expression under red LED light irradiation (Figure 3D,F-H). Likewise, the highest expression of HvFAR6 was observed under blue LED irradiation. It is clear that HvFAR3 is possibly involved in hexacosanol biosynthesis in barley sprouts.

\subsection{Influence of LED-Light Irradiation on Seedling Growth}

In most of the LED treatments in wheat seedlings, there were no statistically significant differences in leaf length, compared to control (FL) seedlings (Figure 4). However, the root growth of wheat sprouts treated with LED light irradiation for 3 days was significantly reduced, compared to that of the controls. Interestingly, further LED treatment on 5 and 7 days did not produce a significant impact on root growth, except under blue LED irradiation on day 7. Nonetheless, continuing white and red LED irradiation up to 9 days reduced root growth, which was statistically significant. In terms of light quality, the highest reduction in root growth was observed under white LED irradiation. Like wheat sprouts, the root growth in barley sprouts was altered by specific light qualities, in accordance with the growth period. Red LED irradiation consistently reduced barley root growth across all growth periods. White or blue LED irradiation also resulted in root growth reduction in one or more growth periods. Although initial treatment with red LED irradiation (on day 3) reduced leaf growth in barley sprouts, the extending the irradiation for 7 or 9 days had a positive impact on barley leaf growth. LED treatment in wheat sprouts showed that the leaf growth was mostly not altered. 

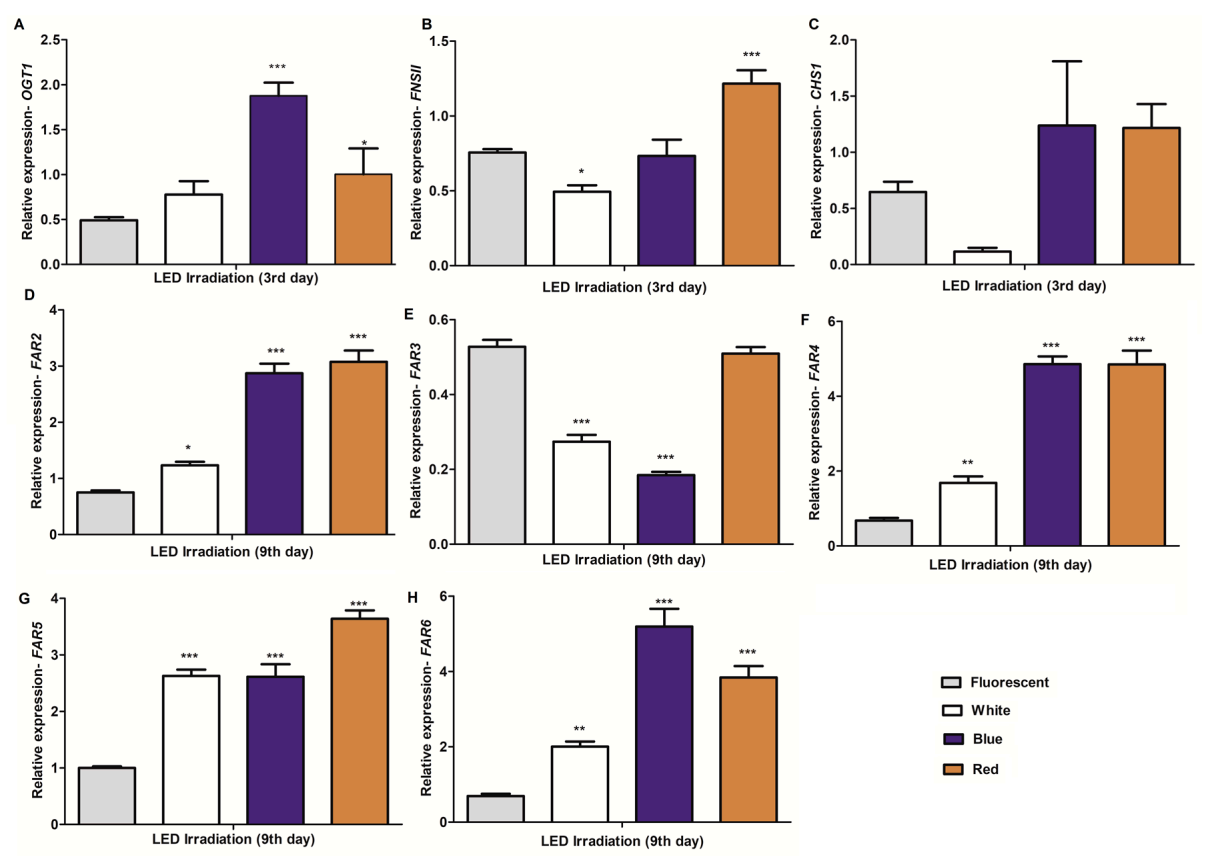

Figure 3. Relative quantification of expression changes in flavonoid and policosanol biosynthesis-related genes in barley sprouts. $(\mathbf{A}-\mathbf{C})$ represent the relative expression levels of UDP-Glc: Isovitexin 7-O-glucosyltransferase 1 (OGT1), flavone synthase II (FNSII), and chalcone synthase 1 (CHS1), respectively. Likewise, (D-H) represent the expression patterns of different classes of fatty acyl-coenzyme A reductase (FAR) genes. The results represent the qRT-PCR-based relative quantification of genes in barley sprouts exposed to fluorescent and LED (white, blue, and red) light irradiations. The gene expression was normalized using the internal control HvActin. ${ }^{*}(p<0.05),{ }^{* *}(p<0.001)$, and ${ }^{* * *}(p<0.0001)$ indicate the statistical significance.
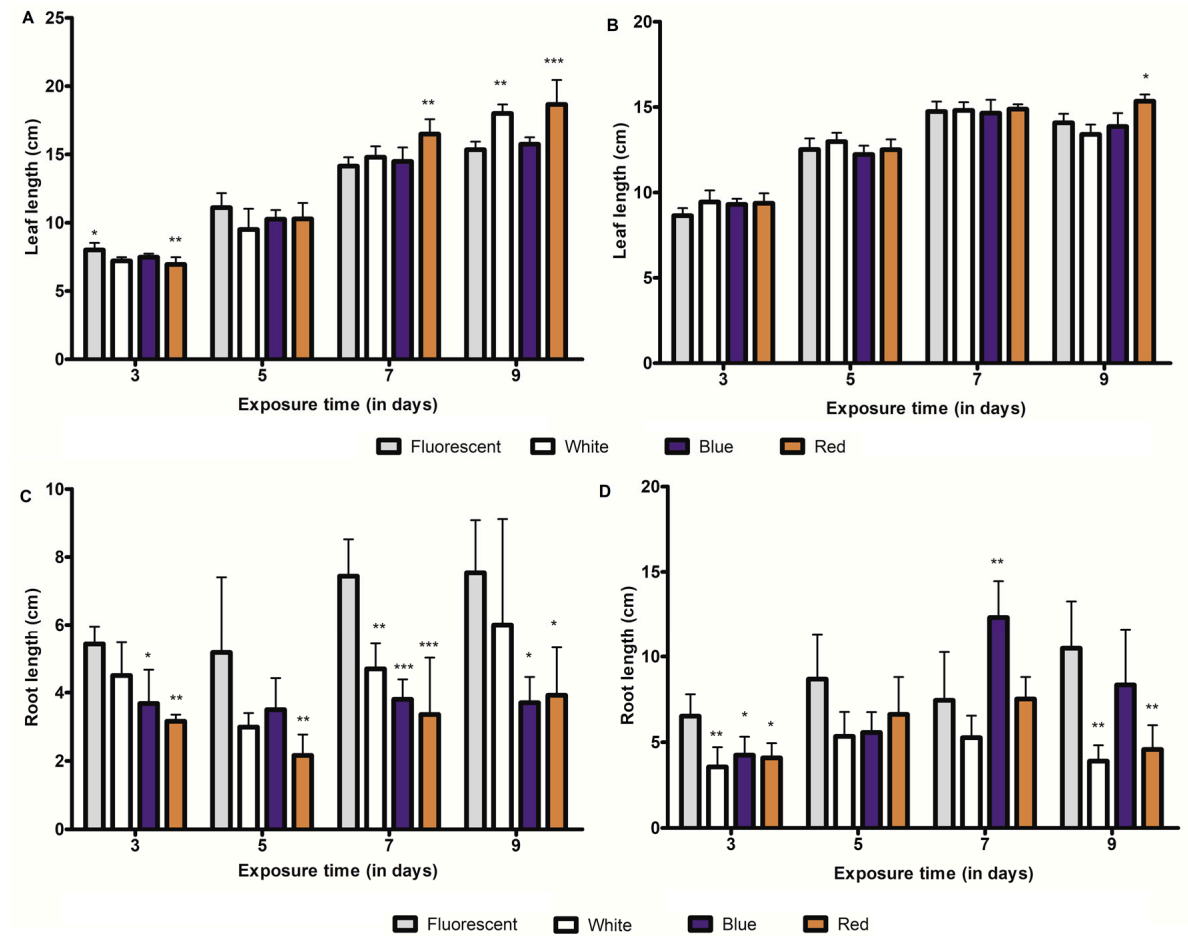

Figure 4. Leaf and root growth parameters of fluorescent and light-emitting diode (LED) light (white, blue, and red) irradiated barley and wheat seedlings at different growth periods. (A,C) represent barley growth parameters, while $(\mathbf{B}, \mathbf{D})$ represent the growth parameters of wheat sprouts. ${ }^{*}(p<0.05)$, ** $(p<0.001)$, and ${ }^{* *}(p<0.0001)$ indicate the statistical significance. 


\section{Discussion}

Phytochromes and cryptochromes are the specialized photoreceptors of plants that sense the spectral quality and quantity, transducing the light signal to regulate genes responsible for secondary metabolite production [30]. Therefore, it is possible to determine the metabolic composition or to enhance the nutritional functionality of the target crops through selective application of light resources and photoperiodism. The application of LEDs for special metabolite production is considered promising, where it has been shown that the metabolic profiles also depend on several other factors, including crop genetics [18,26,31]. The increasing application of LED irradiation sources for the development of designer foods/functional foods may revolutionize the food industry. In this study, we attempted to enhance the $C$-glycosylated flavones/flavonoids and policosanol contents in barley and wheat sprouts using varying light qualities.

C-glycosylated flavones constitute the major portion of flavonoids found in barley seedlings [32]. Saponarin (isovitexin-7-O-glucoside) is a major C-glycosylated flavone, which is naturally present in young barley seedlings [11]. Among cellular organelles, saponarin is efficiently stored in vacuoles [33] and high accumulation is typically observed in primary leaves [4]. Similarly, isoorientin and isoschaftoside are the major C-glycosylated flavones often reported in wheat seedlings [29]. These $C$-glycosylflavones have potential roles as beneficial flavonoids in the human diet $[4,8,34]$. In this study, we found that the $C$-glycosylflavone (saponarin, isoorientin, and isoschaftoside) content was high in the early growth stages of seedlings, where the maximum accumulation was induced by blue LED light irradiation. We found an inverse relationship between $C$-glycosylated flavone content and growth times, indicating that the $C$-glycosylflavone content remains high in young sprouts. In a previous study investigating barley sprouts (13-56 days post-sprouting), it was stated that the saponarin content continued to decline with increasing growth periods [9]. Now, it is clear that the saponarin content in barley sprouts starts declining just three days post-sprouting. In terms of light quality, blue LED light had a positive impact on saponarin content. Blue LED light showed the highest accumulation $(57.7 \%$ and $68.68 \%$ than that in the control, respectively) in barley and wheat sprouts. On the other hand, the impact of red LED light irradiation on saponarin content in barley, and isoorientin and isoschaftoside in wheat sprouts, seemed to differ. In barley, the saponarin content was reduced, while the levels of isoorientin and isoschaftoside were increased in wheat sprouts, suggesting that the effect of red LED light is specific to metabolites and/or crops.

Policosanol (PC) is another beneficial metabolite in the human diet, which is frequently found in cuticular waxes in primary leaves of young cereal sprouts. It represents a mixture of long-chain fatty alcohols (20-36 carbon) mostly comprised of docosanol (C22), tetracosanol (C24), hexacosanol (C26), octacosanol (C28), and triacontanol (C30) [35,36]. Octacosanol and policosanol (long-chain saturated fatty alcohols) are useful in preventing high-fat diet-induced obesity [37]. Owing to its importance in lowering blood cholesterol and protection from platelet aggregation, it has been commercialized in the health industry for a long time [35]. Among the PCs, hexacosanol (C26) and octacosanol (C28) are often observed in barley and wheat sprouts [36,38]. LED light irradiation showed a differential influence on the policosanol content in barley and wheat sprouts, suggesting that the LED response is likely specific to either metabolites or crops. Compared to FL conditions, LED light irradiation reduced the hexacosanol content in barley, while a similar condition in wheat sprouts showed an irregular pattern of octacosanol accumulation; suggesting that factors other than light quality also influence its content in wheat sprouts. Interestingly, in most cases, the policosanol content in barley and wheat sprouts gradually increased with the extension of growth time. Statistical analyses confirmed that the saponarin content under LED treatment was negatively correlated with barley growth periods. A similar negative relationship between content and growth period was also evident in wheat sprouts, albeit restricted to white and/or red LED light treatments. Unlike $C$-glycosylflavone, the hexacosanol content in sprouts appeared to have a positive correlation with sprout growth periods, suggesting the importance of growth level in the determination of policosanol content in barley. Our study corroborates previous findings which have reported a positive correspondence between hexacosanol 
and growth periods in barley [36]. However, a similar correlation pattern was not observed between octacosanol content and growth periods in FL, white, and blue LED irradiated wheat sprouts, possibly suggesting that this relationship might be specific to crop genetics. Altogether, our results show that light qualities and growth periods are two crucial factors in determining the C-glycosylflavone and policosanol contents in barley and wheat sprouts. In addition, light conditions are important parameters in determining the photomorphogenesis of plants [39]. Herein, we found that red LED light irradiation mostly reduced the root growth of sprouts, while white and blue LED light mediated root growth inconsistently across growth periods, indicating the role of other factors regulating growth parameters. The LED light responses of leaves of young seedlings also indicated that LED irradiation does not induce a regular growth pattern for leaves in barley and wheat sprouts. At this stage, it is difficult to conclude the growth impact of LED light irradiation, as this study utilized low intensity LED spectra. Further studies concerning the selection of optimum light intensity and LED spectra for enhancing sprout growth are, therefore, essential.

An interesting observation is that comparison of the metabolite accumulation trends revealed the C-glycosylflavones and policosanol contents to have an inverse relationship in barley sprouts. It is unknown whether this is due to a balancing act of metabolic pathways or just an influencing act of sprout growth periods. It warrants further in-depth studies, in order to understand the underlying mechanisms of metabolite biosynthesis. The molecular basis for the specialized metabolite accumulation to a particular physiological condition is mainly due to changes in the expression pattern of one or more specialized biosynthetic genes. Lee et al. [11] suggested that the expression pattern of UDP-Glc: Isovitexin 7-O-glucosyltransferase (OGT) is likely responsible for saponarin biosynthesis in barley. OGT is responsible for the conversion of isovitexin to saponarin in barley [33]. There are two classes of OGT (OGT1 and OGT2) found in the barley genome, where a change in the expression level of OGT1 has been associated with saponarin concentration [11]. In the present study, we found that a positive correlation exists between the HvOGT1 expression pattern and saponarin accumulation, suggesting the possibility of OGT1 involvement in saponarin biosynthesis. Both metabolite accumulation and HvOGT1 expression were found to be highest after 3 days of treatment. It is clear that blue LED light irradiation accumulated saponarin by upregulating HvOGT1 expression in barley sprouts. The flavonoid biosynthesis pathway gene, HvCHS1, is not linked with saponarin accumulation, indicating the possibility of a specific pathway controlling saponarin biosynthesis in barley. Studies on other crops have shown that fatty acyl-coenzyme A reductases (FAR) are involved in long-chain primary alcohol biosynthesis, part of the cuticular waxes found in leaf surfaces [40]. Studies have also shown that the number of $F A R$ genes and their functions may vary, according to species-specific genetics. In other crops, it is evident that FAR2 and FAR3 are responsible for the biosynthesis of $C_{26}$ and $C_{28}$ primary alcohols [41]. Another report has claimed that at least five FARs are responsible for primary alcohol $\left(\mathrm{C}_{16}\right.$ to $\left.\mathrm{C}_{28}\right)$ biosynthesis in Aegilops tauschii [42]. Until recently, there has been no information about HvFARs and their potential role in hexacosanol biosynthesis. Identification of the FARs responsible for policosanol biosynthesis is inevitable for tailoring metabolic pathways towards enhanced production. Hence, we used the homology-based gene identification method to predict the gene sequences of HvFAR2, HvFAR3, HvFAR4, HvFAR5, and HvFAR6 from available barley genome information. We also measured their expression changes using quantitative PCR during differential light treatments and growth periods, in order to infer their relationship to hexacosanol biosynthesis. Of all the HvFARs analyzed in this study, the expression changes of HvFAR3 were positively associated with hexacosanol accumulation, suggesting their involvement in hexacosanol biosynthesis. Other HvFARs did not have a positive correlation with hexacosanol content, suggesting that they may not be associated with its biosynthesis. In this study, we identified the potential candidate genes involved in saponarin and hexacosanol biosynthesis. These genes can be effectively used to enhance the metabolite concentration by means of genetic manipulation. We also provided an expression atlas of HvFARs during LED light irradiation treatment in sprouts, which may be useful in future studies associated with other policosanol biosynthesis routes. A system-wide 
identification and characterization would add more information about the genetic factors controlling metabolite biosynthesis in barley sprouts.

To conclude, we showed that light qualities and growth times are crucial factors determining the contents of $C$-glycosylflavones and policosanols in barley and wheat sprouts. Blue LED light may be useful for increasing the $C$-glycosylflavone contents in cereal sprouts. Regardless of the light quality, management of growth time of sprouts is essential for policosanol content. This study will help to maximize the beneficial flavonoids and policosanol contents in cereal sprouts through LED applications in the future.

\section{Materials and Methods}

\subsection{Plant Materials, Growth Condition, and LED Treatment}

The barley (Hordeum vulgare L. variety "Keunalbori No.1") and wheat (Triticum asetivum L. variety "Baekchalmil") seeds used by this study were sourced from the National Institute of Crop Science (Korea). The seeds at full maturity were separated by soaking thrice in tap water. Then, visually healthy seeds were once again soaked separately in distilled water for one day. For germination, seeds were sown on 16 plastic sprout cultivating trays $(90 \mathrm{~cm}$ by $30 \mathrm{~cm} ; 180 \mathrm{~g}$ per tray) and maintained in greenhouse conditions $\left(25^{\circ} \mathrm{C}, 16 \mathrm{~h}\right.$ photoperiod) for one day. Four trays with sprouting seeds (per treatment) were moved to individual growth chambers (Mokmin Co., Ltd, Suwon, South Korea) equipped with FL or different spectra LED light irradiations ( $470 \mathrm{~nm}$ for blue, $380 \mathrm{~nm}$ for white, and $660 \mathrm{~nm}$ for red) over 9 days (Figure 5). Sprouts were watered at regular intervals. The photosynthetic photon flux density (PPFD) of FL $\left(96 \mu \mathrm{mol} \mathrm{m}^{-2} \mathrm{~s}^{-1}\right)$, blue $\left(100 \mu \mathrm{mol} \mathrm{m}^{-2} \mathrm{~s}^{-1}\right)$, white $\left(100 \mu \mathrm{mol} \mathrm{m}^{-2} \mathrm{~s}^{-1}\right)$, and red $\left(93 \mu \mathrm{mol} \mathrm{m}^{-2} \mathrm{~s}^{-1}\right)$ lights was measured at plant level by using a quantum meter (Apogee Instruments, Logan, USA). Fresh leaves and coleoptiles of each treatment were harvested simultaneously at 3, 5, 7, and 9 days after irradiation (DAI). All samples were frozen in liquid nitrogen and stored at $-80^{\circ} \mathrm{C}$ for RNA extraction and metabolomic analyses. All sprouting seeds throughout the treatments were maintained in a growth chamber with $16 \mathrm{~h}$ photoperiods, $60-80 \%$ humidity, and $22-25^{\circ} \mathrm{C}$.

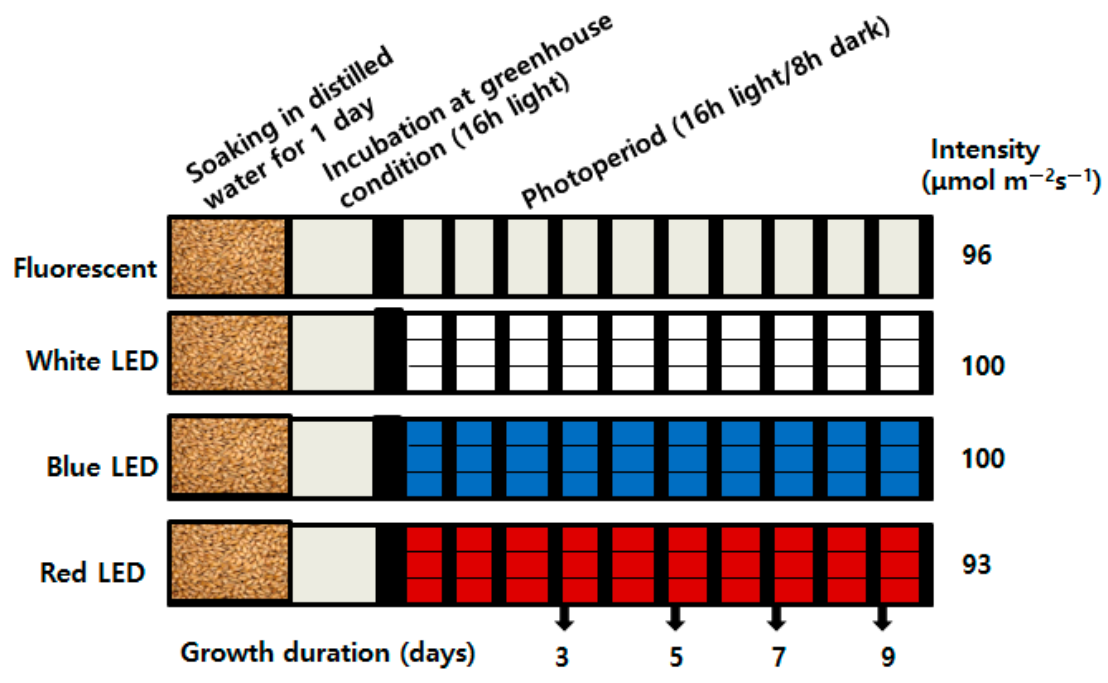

Figure 5. Schematic representation of experimental design used for LED treatment on barley and wheat seedlings. The diagram illustrates the germination method, growth period, and light quality and intensity.

\subsection{Extraction of C-glycosylated Flavones and Measurement}

The extraction method of Lee et al. [11] was used for the preparation of crude extracts from dried barley and wheat seedlings. An ultra-high-performance liquid chromatograph equipped with UV detectors (Dionex Ultimate 3000; Thermo Scientific, Waltham, MA, USA) and reversed-phase 
HPLC column (ACQUITY UPLC BEH C18, $2.1 \mathrm{~mm}$ by $100 \mathrm{~mm}$ ) was utilized for separation and quantitative analyses [8]. One gram of freeze-dried and chopped seedlings from each treatment was extracted by treating with either $50 \%$ ethanol (barley) or $100 \%$ methanol (wheat) on a shaker for $24 \mathrm{~h}$ at room temperature. The ethanolic or methanolic extracts were then filtered through $0.2 \mu \mathrm{m}$ syringe filters. Following evaporation under vacuum, the extracts were dissolved in $10 \%$ dimethyl sulfoxide (DMSO) containing 50\% ethanol and $1.3 \mu \mathrm{L}$ was injected into the column for separation and detection at $325 \mathrm{~nm}$. The mobile phase was comprised of trifluoroacetic acid (TFA) $(0.1 \%)$ in water (A) and acetonitrile (B) with a flow rate of $0.5 \mathrm{~mL} / \mathrm{min}$, which was applied for separation of the analyte. Saponarin, isoorientin, and isoschaftoside molecules were identified by comparing the retention times to those of standards (obtained from Extrasynthese, Lyon, France and NICS, Jeonju, Korea). A standard calibration curve was prepared by plotting the peak area (y) of the chromatogram and the respective concentrations $(31.25,62.5,125,250,500$, and $1000 \mu \mathrm{g} / \mathrm{mL})(\mathrm{x})$. The equations of the calibration curves for saponarin, isoorientin, and isoschaftoside were $y=0.0876 \mathrm{x}+0.3514\left(\mathrm{r}^{2}=0.999\right)$, $y=0.0917 x-0.5536\left(r^{2}=0.998\right)$, and $y=0.0 .0547 x+0.22935\left(r^{2}=0.999\right)$, respectively. The metabolite content $(\mathrm{mg} / \mathrm{g}$ DW) from three technical replicates of biologically independent samples were used as input for the statistical analyses.

\subsection{Extraction and Quantification of Policosanols from Barley and Wheat Seedlings}

The preparation of barley and wheat crude extracts, policosanol standards, GC/MS parameters, and the quantification method of GC/MS as described elsewhere [17,36], was used in this study. Briefly, $1 \mathrm{~g}$ of freeze-dried and chopped samples collected from all treatments was extracted separately into $10 \mathrm{~mL}$ of hexane on a shaker for $24 \mathrm{~h}$ at room temperature. The supernatant of the mixture was collected by centrifugation at $3000 \mathrm{~g}$ for $3 \mathrm{~min}$, filtered through a syringe filter with a pore size of $0.45 \mu \mathrm{m}$ (Whatman Inc., Maidstone, UK), and kept under vacuum conditions until the hexane was completely removed. To the final extract, $250 \mu \mathrm{L}$ of N-Methyl-N-trimethylsilyfluoroacetamide (MSTFA) and $0.5 \mathrm{~mL}$ of chloroform was added and stirred for $15 \mathrm{~min}$ at $60^{\circ} \mathrm{C}$. Chloroform was added to make up one $\mathrm{ml}$ of sample and, then, one $\mu \mathrm{L}$ was injected into the gas chromatograph using an auto sampler with a split ratio of 1:5. The GC was equipped with an HP-5MS UI (diphenyl 5\%-dimethylsiloxane $95 \%$ co-polymer) capillary column ( $30 \mathrm{~m}$ by $0.25 \mu \mathrm{m}$ by $0.25 \mu \mathrm{m}$ film thickness) and a 5977A series mass spectroscopy (Agilent Technologies, Palo Alto, CA). The policosanol content was quantified according to the methods of Ra et al. [17]. The policosanol standards-Eicosanol $\left(\mathrm{C}_{20}\right)$, heneicosanol $\left(C_{21}\right)$, docosanol $\left(C_{22}\right)$, tricosanol $\left(C_{23}\right)$, tetracosanol $\left(C_{24}\right)$, hexacosanol $\left(C_{26}\right)$, heptacosanol $\left(C_{27}\right)$, octacosanol $\left(\mathrm{C}_{28}\right)$, and triacontanol $\left(\mathrm{C}_{30}\right)$-hexane, and chloroform solutions were purchased from Sigma (Sigma-Aldrich, St. Louis, MO).

\subsection{Identification of Saponarin and Policosanol Biosynthetic-Related Genes}

In general, flavone/flavonoid and long-chain fatty alcohol (or primary alcohol) biosynthesis pathways comprised of one or more enzymes and their information in barley are limited. Hence, the gene names and sequence information of other crops were searched for sequence homologies to unearth orthologous transcripts (full and partial) in Hordeum vulgare and Triticum asetivum species. The gene sequences were retrieved from NCBI Gene [43], PlantsDB [44], and GrainGenes [45] databases. For annotation, potential orthologous gene sequences were searched for the presence of conserved domains using the NCBI Conserved Domains [46] database. Transcripts encoding for flavone/flavonoid-related-UDP-Glc: OGT1, flavone synthase II (FNSII), and chalcone synthase 1 (CHS1) - and fatty alcohol-related - fatty acyl-CoA reductase 2 (FAR2), FAR3, FAR4, FAR5, and FAR6biosynthesis were identified in barley. The designated gene sequences are listed in Table $\mathrm{S} 1$, which were utilized in primer synthesis for qRT-PCR-based gene expression profiling. 


\subsection{Analysis of Expression Pattern of Biosynthetic Related Genes in Seedlings Subjected to LED Light Irradiation}

Approximately $100 \mathrm{mg}$ of powdered leaf samples of five or more seedlings were utilized for total RNA extraction using an RNeasy Plant Mini kit (Qiagen, Hilden, Germany). First-strand cDNA was synthesized from DNA decontaminated total RNA $(5 \mu \mathrm{g})$ of each treatment using amfiRivert cDNA Synthesis Platinum Master mix (GenDepot, Katy, TX, USA). The diluted cDNA (1:10) was used as a template, along with gene-specific primers (Table S1) and AccuPower $2 \times$ GreenStar Master Mix (Bioneer, Daejeon, Korea), for relative quantification of saponarin, policosanol, and general flavonoid biosynthesis-related genes in quantitative RT-PCR with CFX96 ${ }^{\mathrm{TM}}$ Real-Time PCR Detection System (Bio-Rad, Hercules, CA, USA). HoActin was used as an internal control. The relative quantification of gene expression was calculated using the $2^{-\Delta \Delta C T}$ method.

\subsection{Phenotyping of LED-Treated Cereal Sprouts}

The effect of LED light irradiation on the growth of wheat and barley sprouts was evaluated from five or more seedlings of each treatment, collected after 3-9 days of treatment. The length of all primary leaves and roots were measured. The mean values of growth parameters were compared between treatments and the mean differences were evaluated by statistical significance analysis.

\subsection{Statistical Analyses}

All treatments and experiments in this study had at least three independent biological and technical replicates. The mean values are presented in graphs drawn using the GraphPad Prism 5 software, while standard deviations are represented as error bars. One-way analysis of variance (one-way ANOVA) and Tukey's HSD test were carried out to assess the significant variations existing between the treatments and analyses performed in this study. Statistics by ANOVA test are shown; ${ }^{*} p<0.05,{ }^{* *} p<0.001$, and ${ }^{* * *} p<0.0001$. Pearson correlation coefficients and their significance were measured using the Social Science Statistics [47].

Supplementary Materials: The following are available online at http://www.mdpi.com/2223-7747/9/11/1502/s1, Figure S1: Representative chromatogram of saponarin and hexacosanol of barley seedlings exposed to differential LED light treatments. Figure S2: Chromatogram of isoorientin, isoschaftoside, and octacosanol of wheat seedlings exposed to differential LED light treatments. Figure S3: Effect of growth periods on the C-glycosylflavone and policosanol contents in fluorescent and LED light irradiated barley and wheat sprouts. Table S1: List of genes, respective gene and protein IDs, primer sequences, and their annealing temperatures used in the quantitative RT-PCR assay.

Author Contributions: Conceptualization, S.I.L.; investigation, M.M., J.H.K., S.H.K., J.Y.K., J.W.H., H.L., K.-S.L., W.D.S., S.P., J.A.K.; writing-original draft preparation, M.M.; writing-review and editing, M.M. and S.I.L. All authors have read and agreed to the published version of the manuscript.

Funding: This research was funded by the Rural Development Administration (Korea) through the Rural Program for Agricultural Science and Technology Development, grant number PJ01495701 and Cooperative Research Program for Agricultural Science and Technology, grant number PJ01421201.

Conflicts of Interest: The authors declare that they have no conflict of interest.

\section{References}

1. Giraldo, P.; Benavente, E.; Manzano-Agugliaro, F.; Gimenez, E. Worldwide research trends on wheat and barley: A bibliometric comparative analysis. Agronomy 2019, 9, 352. [CrossRef]

2. Shewry, P.R.; Hey, S.J. The contribution of wheat to human diet and health. Food Energy Secur. 2015, 4, 178-202. [CrossRef] [PubMed]

3. Byun, A.R.; Chun, H.; Lee, J.; Lee, S.W.; Lee, H.S.; Shim, K.W. Effects of a dietary supplement with barley sprout extract on blood cholesterol metabolism. Evid. Based Complement. Altern. Med. 2015, 473056, 1-7. [CrossRef] [PubMed]

4. Zeng, Y.; Pu, X.; Yang, J.; Du, J.; Yang, X.; Li, X.; Li, L.; Zhou, Y.; Yang, T. Preventive and therapeutic role of functional ingredients of barley grass for chronic diseases in human beings. Oxid. Med. Cell. Longev. 2018, 2018, 3232080. [CrossRef] [PubMed] 
5. Lee, J.H.; Jia, Y.; Thach, T.T.; Han, Y.; Kim, B.; Wu, C.; Kim, Y.; Seo, W.D.; Lee, S.J. Hexacosanol reduces plasma and hepatic cholesterol by activation of AMP-activated protein kinase and suppression of sterol regulatory element-binding protein-2 in HepG2 and C57BL/6J mice. Nutr. Res. 2017, 43, 89-99. [CrossRef]

6. Lee, Y.H.; Kim, S.H.; Lee, S.; Kim, K.M.; Jung, J.C.; Son, T.G.; Ki, S.H.; Seo, W.D.; Kwak, J.H.; Hong, J.T.; et al. Antioxidant effect of barley sprout extract via enhancement of Nuclear Factor-Erythroid 2 Related Factor 2 activity and glutathione synthesis. Nutrients 2017, 9, 1252. [CrossRef] [PubMed]

7. Kim, Y.J.; Hwang, S.H.; Jia, Y.; Seo, W.D.; Lee, S.J. Barley sprout extracts reduce hepatic lipid accumulation in ethanol-fed mice by activating hepatic AMP-activated protein kinase. Food Res. Int. 2017, 101, $209-217$. [CrossRef]

8. Seo, K.H.; Park, M.J.; Ra, J.E.; Han, S.I.; Nam, M.H.; Kim, J.H.; Lee, J.H.; Seo, W.D. Saponarin from barley sprouts inhibits NF-kB and MAPK on LPS-induced RAW 264.7 cells. Food Funct. 2014, 5, 3005-3013. [CrossRef]

9. Park, M.J.; Seo, W.D.; Kang, Y.H. The antioxidant properties of four korean barley cultivars at different harvest times and profiling of major metabolites. J. Agric. Sci. 2015, 7, 94. [CrossRef]

10. Seo, W.; Hae, J.; Jia, Y.; Wu, C.; Lee, S. Saponarin activates AMPK in a calcium-dependent manner and suppresses gluconeogenesis and increases glucose uptake via phosphorylation of CRTC2 and HDAC5. Bioorg. Med. Chem. Lett. 2015, 25, 5237-5242. [CrossRef]

11. Lee, H.; Ra, S.W.J.; Woo, K.L.; Seo, D.; Hwan, J. Saponarin content and biosynthesis-related gene expression in young barley (Hordeum vulgare L.) seedlings. J. Plant Biotechnol. 2019, 2818, 247-254. [CrossRef]

12. Ye, T.; Su, J.; Huang, C.; Yu, D.; Dai, S.; Huang, X.; Chen, B.; Zhou, M. Isoorientin induces apoptosis, decreases invasiveness, and downregulates VEGF secretion by activating AMPK signaling in pancreatic cancer cells. OncoTargets Targets 2016, 9, 7481-7492. [CrossRef]

13. Xiao, J.; Capanoglu, E.; Jassbi, A.R.; Miron, A. Advance on the flavonoid C-glycosides and health benefits. Crit. Rev. Food Sci. Nutr. 2016, 56, S29-S45. [CrossRef]

14. Nam, T.G.; Kim, D.-O.; Eom, S.H. Effects of light sources on major flavonoids and antioxidant activity in common buckwheat sprouts. Food Sci. Biotechnol. 2018, 27, 169-176. [CrossRef] [PubMed]

15. Sytar, O.; Bosko, P.; Živčák, M.; Brestic, M.; Smetanska, I. Bioactive phytochemicals and antioxidant properties of the grains and sprouts of colored wheat genotypes. Molecules 2018, 23, 2282. [CrossRef]

16. Benincasa, P.; Falcinelli, B.; Lutts, S.; Stagnari, F.; Galieni, A. Sprouted grains: A comprehensive review. Nutrients 2019, 11, 421. [CrossRef] [PubMed]

17. Ra, J.; Woo, S.; Lee, K.; Ja, M.; Young, H.; Mi, H.; Chung, I.; Hyun, D.; Hwan, J.; Duck, W. Policosanol profiles and adenosine $5^{\prime}$-monophosphate-activated protein kinase (AMPK) activation potential of Korean wheat seedling extracts according to cultivar and growth time. Food Chem. 2020, 317, 126388. [CrossRef] [PubMed]

18. Lemmens, E.; Moroni, A.V.; Pagand, J.; Heirbaut, P.; Ritala, A.; Karlen, Y.; Kim-Anne, L.; Van den Broeck, H.C.; Brouns, F.J.P.H.; De Brier, N.; et al. Impact of cereal seed sprouting on its nutritional and technological properties: A Critical Review. Compr. Rev. Food Sci. Food Saf. 2019, 18, 305-328. [CrossRef]

19. Rico, D.; Peñas, E.; del Carmen García, M.; Martínez-Villaluenga, C.; Rai, D.K.; Birsan, R.I.; Frias, J.; Martín-Diana, A.B. Sprouted barley flour as a nutritious and functional ingredient. Foods 2020, 9, 296. [CrossRef] [PubMed]

20. Aborus, N.E.; Canadanovi, J.; Saponjac, V.T. Powdered barley sprouts: Composition, functionality and polyphenol digestibility. Int. J. Food Sci. Technol. 2017, 52, 231-238. [CrossRef]

21. Wu, X.; Cai, K.; Zhang, G.; Zeng, F. Metabolite profiling of barley grains subjected to water stress: To explain the genotypic difference in drought-induced impacts on malting quality. Front. Plant. Sci. 2017, 8, 1547. [CrossRef]

22. Wang, Y.; Zeng, X.; Xu, Q.; Mei, X.; Yuan, H.; Jiabu, D.; Sang, Z.; Nyima, T. Metabolite profiling in two contrasting Tibetan hulless barley cultivars revealed the core salt-responsive metabolome and key salt-tolerance biomarkers. AoB Plants 2019, 11, plz021. [CrossRef]

23. Kang, C.H.; Yoon, E.K.; Muthusamy, M.; Kim, J.A.; Jeong, M.J.; Lee, S.I. Blue LED light irradiation enhances L-ascorbic acid content while reducing reactive oxygen species accumulation in Chinese cabbage seedlings. Sci. Hortic. 2020, 261, 108924. [CrossRef]

24. Cheng, C.; Liu, Y.; Fang, W.; Tao, J.; Yang, Z.; Yin, Y. iTRAQ-based proteomic and physiological analyses of mustard sprouts in response to heat stress. RSC Adv. 2020, 10, 6052-6062. [CrossRef] 
25. Muthusamy, M.; Hwang, J.E.; Kim, S.H.; Kim, J.A.; Jeong, M.J.; Park, H.C.; Lee, S.I. Elevated carbon dioxide significantly improves ascorbic acid content, antioxidative properties and restricted biomass production in cruciferous vegetable seedlings. Plant. Biotechnol. Rep. 2019, 13, 293-304. [CrossRef]

26. Tuan, P.A.; Thwe, A.A.; Kim, Y.B.; Kim, J.K.; Kim, S.J.; Lee, S.; Chung, S.O.; Park, S.U. Effects of white, blue, and red light-emitting diodes on carotenoid biosynthetic gene expression levels and carotenoid accumulation in sprouts of tartary buckwheat (Fagopyrum tataricum gaertn.). J. Agric. Food Chem. 2013, 61, 12356-12361. [CrossRef]

27. Meng, T.; Nakamura, E.; Irino, N.; Joshi, K.R.; Devkota, H.P.; Yahara, S.; Kondo, R. Effects of irradiation with light of different photon densities on the growth of young green barley plants. Agric. Sci. 2015, 06, 208-216. [CrossRef]

28. Hasan, M.M.; Bashir, T.; Bae, H. Use of utrasonication tchnology for the icreased poduction of pant scondary mtabolites. Molecules 2017, 22, 1046. [CrossRef]

29. Kowalska, I.; Jedrejek, D.; Jonczyk, K.; Stochmal, A. UPLC-PDA-ESI-MS analysis and TLC-DPPH activity of wheat varieties. Acta Chromatogr. 2019, 31, 151-156. [CrossRef]

30. Kopsell, D.A.; Sams, C.E. Increases in shoot tissue pigments, glucosinolates, and mineral elements in sprouting broccoli after exposure to short-duration blue light from light emitting diodes. J. Am. Soc. Hortic. Sci. 2013, 138, 31-37. [CrossRef]

31. Ghimire, B.K.; Yu, C.Y.; Chung, I.M. Assessment of the phenolic profile, antimicrobial activity and oxidative stability of transgenic Perilla frutescens L.overexpressing tocopherol methyltransferase $(\gamma$-tmt) gene. Plant. Physiol. Biochem. 2017, 118, 77-87. [CrossRef]

32. Brauch, D.; Porzel, A.; Schumann, E.; Pillen, K.; Mock, H.P. Changes in isovitexin-O-glycosylation during the development of young barley plants. Phytochemistry 2018, 148, 11-20. [CrossRef]

33. Marinova, K.; Kleinschmidt, K.; Weissenböck, G.; Klein, M. Flavonoid biosynthesis in barley primary leaves requires the presence of the vacuole and controls the activity of vacuolar flavonoid transport. Plant. Physiol. 2007, 144, 432-444. [CrossRef]

34. Kamiyama, M.; Shibamoto, T. Flavonoids with potent antioxidant activity found in young green barley leaves. J. Agric. Food Chem. 2012, 60, 6260-6267. [CrossRef]

35. Irmak, S.; Dunford, N.T.; Milligan, J. Policosanol contents of beeswax, sugar cane and wheat extracts. Food Chem. 2006, 95, 312-318. [CrossRef]

36. Seo, W.D.; Yuk, H.J.; Curtis-long, M.J.; Jang, K.C.; Lee, J.H.; Han, S.; Kang, H.W.; Nam, M.H.; Lee, S.; Lee, J.H.; et al. Effect of the growth stage and cultivar on policosanol profiles of barley sprouts and their adenosine 5'-monophosphate-activated protein kinase activation. J. Agric. Food Chem. 2013, 61, 1117-1123. [CrossRef]

37. Sharma, R.; Matsuzaka, T.; Kaushik, M.K.; Sugasawa, T.; Ohno, H.; Wang, Y.; Motomura, K.; Shimura, T.; Okajima, Y.; Mizunoe, Y.; et al. Octacosanol and policosanol prevent high-fat diet-induced obesity and metabolic disorders by activating brown adipose tissue and improving liver metabolism. Sci. Rep. 2019, 9, 5169. [CrossRef]

38. Richardson, A.; Franke, R.; Kerstiens, G.; Jarvis, M.; Schreiber, L.; Fricke, W. Cuticular wax deposition in growing barley (Hordeum vulgare) leaves commences in relation to the point of emergence of epidermal cells from the sheaths of older leaves. Planta 2005, 222, 472-483. [CrossRef]

39. Gil, K.E.; Park, C.M. Thermal adaptation and plasticity of the plant circadian clock. New Phytol. 2019, 221, 1215-1229. [CrossRef]

40. Wang, Y.; Wang, M.; Sun, Y.; Wang, Y.; Li, T.; Chai, G.; Jiang, W.; Shan, L.; Li, C.; Xiao, E.; et al. FAR5, a fatty acyl-coenzyme A reductase, is involved in primary alcohol biosynthesis of the leaf blade cuticular wax in wheat (Triticum aestivum L.). J. Exp. Bot. 2015, 66, 1165-1178. [CrossRef]

41. Wang, Y.; Sun, Y.; You, Q.; Luo, W.; Wang, C.; Zhao, S.; Chai, G.; Li, T.; Shi, X.; Li, C.; et al. Three Fatty Acyl-Coenzyme A Reductases, BdFAR1, BdFAR2 and BdFAR3, are involved in cuticular wax primary alcohol biosynthesis in Brachypodium distachyon. Plant. Cell Physiol. 2018, 59, 527-543. [CrossRef]

42. Wang, M.; Wu, H.; Xu, J.; Li, C.; Wang, Y.; Wang, Z. Five fatty acyl-coenzyme a reductases are involved in the biosynthesis of primary alcohols in Aegilops tauschii leaves. Front. Plant Sci. 2017, 8, 1012. [CrossRef]

43. NCBI Gene. Available online: https://www.ncbi.nlm.nih.gov/gene (accessed on 7 August 2020).

44. PlantsDB. Available online: http://pgsb.helmholtz-muenchen.de/plant/search.jsp (accessed on 7 August 2020).

45. GrainGenes. Available online: https://wheat.pw.usda.gov/blast/ (accessed on 7 August 2020). 
46. NCBI Conserved Domains. Available online: https://www.ncbi.nlm.nih.gov/Structure/cdd/wrpsb.cgi (accessed on 8 August 2020).

47. Social Science Statistics. Available online: https://www.socscistatistics.com/tests/pearson/default.aspx. (accessed on 26 October 2020).

Publisher's Note: MDPI stays neutral with regard to jurisdictional claims in published maps and institutional affiliations.

(C) 2020 by the authors. Licensee MDPI, Basel, Switzerland. This article is an open access article distributed under the terms and conditions of the Creative Commons Attribution (CC BY) license (http://creativecommons.org/licenses/by/4.0/). 